\title{
Genetic correlations between measures of Mycobacterium bovis infection and economically important traits in Irish Holstein-Friesian dairy cows
}

\author{
M. L. Bermingham, ${ }^{* 1}$ S. J. More,$\dagger$ M. Good, $\ddagger$ A. R. Cromie,§ I. M. Higgins, $†$ and D. P. Berry* \\ *Moorepark Production Research Centre, Fermoy, Co. Cork, Ireland \\ †Centre for Veterinary Epidemiology and Risk Analysis, School of Agriculture, Food Science and Veterinary Medicine, University College Dublin, \\ Belfield 4, Ireland \\ ‡Department of Agriculture, Fisheries and Food, Dublin 2, Ireland \\ $\S$ The Irish Cattle Breeding Federation, Bandon, Co. Cork, Ireland
}

\section{ABSTRACT}

Mycobacterium bovis is the primary agent of tuberculosis (TB) in cattle. The failure of Ireland and some other countries to reach TB-free status indicates a need to investigate complementary control strategies. One such approach would be genetic selection for increased resistance to TB. Previous research has shown that considerable genetic variation exists for susceptibility to the measures of $M$. bovis infection, confirmed $M$. bovis infection, and $M$. bovis-purified protein derivative (PPD) responsiveness. The objective of this study was to estimate the genetic and phenotypic correlations between economically important traits and these measures of $M$. bovis infection. A total of 20,148 and 17,178 cows with confirmed $M$. bovis infection and $M$. bovis-PPD responsiveness records, respectively, were available for inclusion in the analysis. First- to thirdparity milk, fat, and protein yields, somatic cell count, calving interval, and survival, as well as first-parity body condition score records, were available on cows that calved between 1985 and 2007. Bivariate linearlinear and threshold-linear sire mixed models were used to estimate (co)variance components. The genetic correlations between economically important traits and the measures of $M$. bovis infection estimated from the linear-linear and threshold-linear sire models were similar. The genetic correlations between susceptibility to confirmed $M$. bovis infection and economically important traits investigated in this study were all close to zero. Mycobacterium bovis-PPD responsiveness was positively genetically correlated with fat production (0.39) and body condition score (0.36), and negatively correlated with somatic cell score $(-0.34)$ and survival $(-0.62)$. Hence, selection for increased survival may indirectly reduce susceptibility to $M$. bovis infection, whereas selection for reduced somatic cell count and

Received November 19, 2009.

Accepted August 1, 2010.

${ }^{1}$ Corresponding author: Mairead.Bermingham@roslin.ed.ac.uk increased fat production and body condition score may increase susceptibility to $M$. bovis infection.

Key words: dairy cow, genetic correlation, Mycobacterium bovis, tuberculosis

\section{INTRODUCTION}

Tuberculosis (TB) is a chronic bacterial disease in humans and animals characterized by the progressive development of granulomatous lesions or tubercles in diseased tissues (Thoen and Bloom, 1995). In Ireland, a TB eradication policy was introduced in 1954 to reduce the then estimated $17 \%$ animal incidence of bovine TB, according to Department of Agriculture, Fisheries and Food (DAFF) statistics. Progress was rapid during the initial stages of the program, leading to a considerable reduction in disease incidence in cattle by the mid-1960s. However, since then, progress has stalled; animal incidence of TB was $0.33 \%$ in 2004 (Good et al., 2007).

Mycobacterium bovis is the primary agent of TB in cattle. The failure of Ireland, and some other countries, to reach TB-free bovine herd status is due to a range of factors relating to ongoing wildlife-to-cattle and cattleto-cattle transmission (More and Good, 2006). Genetic selection for increased resistance to TB in cattle could hypothetically complement current eradication efforts. Two recent studies have demonstrated that genetic variation exists in abattoir-confirmed $M$. bovis infection (Bermingham et al., 2009; Brotherstone et al., 2010), which indicates that genetic improvement is possible. In Ireland, clinical disease is now rarely seen, rendering post mortem inspections a far less sensitive $M$. bovis infection trait measure (test sensitivity, $47 \%$; Corner et al., 1990) than responsiveness to the single intradermal comparative tuberculin test (SICTT; test sensitivity, 91\%; More and Good, 2006). Significant genetic variation for responsiveness to the SICTT (Bermingham et al., 2009; Brotherstone et al., 2010), and a near unity genetic correlation with susceptibility to confirmed $M$. bovis infection, has been estimated in Irish Holstein- 
Friesian dairy cows (Bermingham et al., 2009), suggesting that indirect selection for increased resistance to confirmed $M$. bovis infection is possible using routinely recorded SICTT data.

A strong negative genetic correlation between milk yield and susceptibility to confirmed $M$. bovis infection has been estimated recently in U.K. dairy cattle, indicating that selection for increased resistance to $M$. bovis infection would not conflict with improving milk yield of lactating dairy cows (Brotherstone et al., 2010). Nevertheless, a clear antagonistic genetic correlation between milk production traits and risk of clinical mastitis has been demonstrated (Heringstad et al., 2005; Negussie et al., 2008). The cell-mediated immune response (CMIR) dominates host response to intracellular parasites, such as $M$. bovis. In contrast, the antibodymediated immune response (AMIR) predominantly aids in protection against extracellular parasites, such as the etiological agent of clinical mastitis, Escherichia coli (Rupp et al., 2007). Selection for mastitis resistance may therefore have a detrimental effect on the genetic efficacy of the CMIR. Furthermore, the estimates of the genetic correlations reported in the literature between susceptibility to diseases other than mastitis are inconsistent (Ingvartsen et al., 2003). Therefore, the effect of selection for economically important traits in Irish dairy cattle on levels of genetic resistance to $M$. bovis infection needs to be investigated.

The objective of the present study was to estimate the genetic correlations between measures of $M$. bovis infection and economically important traits to determine the effect of selection for increased performance on genetic resistance to $M$. bovis infection and to evaluate the feasibility of selection for increased resistance to confirmed $M$. bovis infection in Irish Holstein-Friesian dairy cattle.

\section{MATERIALS AND METHODS}

\section{Data}

A detailed description of the $M$. bovis-purified protein derivative (PPD) responsiveness and confirmed $M$. bovis infection trait measures are described in detail by Bermingham et al. (2009). The edits used to generate the respective datasets are described in the appendix of this paper.

M. bovis Infection Trait Measures. Mycobacterium bovis-PPD responsiveness data were compiled from national SICTT field surveillance data from November 2000 to December 2007. The test involves concurrent injection of $M$. bovis and Mycobacterium avium-PPD antigen (i.e., a measure of sensitization to $M$. avium species and other non-tuberculous Mycobacteria species) into the neck of the animal. The difference in response to the $M$. bovis and $M$. avium antigens is measured $72 \mathrm{~h}$ after injection by subtracting the size of the $M$. avium-PPD response from that of the $M$. bovis-PPD. In the present study, animal $M$. bovis-PPD responsiveness was dichotomized as $M$. bovis standard reactor (i.e., an animal with a skin change in response to the $M$. bovis-PPD inoculation more than $4 \mathrm{~mm}$ greater than the reaction to the $M$. avium-PPD inoculation) or nonreactor (i.e., an animal with a skin change in response to the $M$. bovis-PPD inoculation equal to or less than the reaction to the $M$. avium-PPD inoculation). Inconclusive $M$. bovis reactors (animals with a skin change in response to the $M$. bovis-PPD inoculation larger than $0 \mathrm{~mm}$ but less than $4 \mathrm{~mm}$ greater than the reaction to the $M$. avium-PPD inoculation) were removed from the analysis. The $M$. bovis-PPD responsiveness dataset included in the analysis comprised 17,178 cows within 598 disease outbreaks or episodes from 549 herds.

Confirmed $M$. bovis infection data were compiled from national abattoir postmortem meat inspection TB lesion records from November 2000 to December 2007. Lesions disclosed in nonreactor cattle post-slaughter were confirmed as TB through histopathology or culture by the Central Veterinary Research Laboratory (Dublin, Ireland). Confirmed M. bovis infection was dichotomized as lesioned (i.e., an animal with a confirmed tuberculous lesion) or nonlesioned (i.e., a nonreactor with no subsequent history of $M$. bovis infection). The confirmed $M$. bovis infection dataset included in the analysis comprised 20,148 cows within 647 episodes from 608 herds.

Performance Traits. First- to third-parity 305-d milk, fat, and protein yield; SCC; calving date and calving interval (CIN; defined as the number of days between consecutive calvings); and first-parity BCS records were extracted from the Irish Cattle Breeding Federation database for cows calving between January 1985 and December 2007. The natural logarithm of SCC divided by 1,000 was used to normalize the data; this variable is referred to herein as SCS. Cow BCS was scored on a scale of 1 (anorexic) to 9 (obese). Survival from lactation 1 to 2, 2 to 3 , and 3 to 4 were obtained for each cow. The performance trait dataset included in the analysis comprised 224,922 cows, of which 105,064 had information on production $(57,757$, 51,564, and 24, 216 for first-, second-, and third-parity records, respectively), 112,337 had information on CIN (92,006, 36,097, and 16,237 for first-, second-, and third-parity records, respectively), 95,239 had information on survival $(88,930,41,097$, and 20,744 records for survival from first to second, second to third, and third to fourth parity, respectively), and 57,250 had information on first-parity BCS. All datasets had ap- 
proximately 1,000 cows with both performance and $M$. bovis infection trait measure records, apart from the BCS data, which had 354 cows with both BCS and $M$. bovis infection trait measure records.

\section{Data Analysis}

Linear-linear sire models, which ignored the binary nature of the $M$. bovis infection trait measure data, were used to estimate the (co)variance components among the $M$. bovis infection trait measures and performance traits in ASREML (Gilmour et al., 2009). The fixed effect of Holstein breed proportion, breed proportion other than Holstein-Friesian, heterosis, and recombination loss were adjusted for in all models. The models for measures of $M$. bovis infection included the fixed effects of herd-episode, year of herd-test $\times$ month of test interaction, month of calving, parity, and age (months) nested within parity. All performance trait models were adjusted for the fixed effect of contemporary group and age at calving. The BCS model was adjusted for the fixed effect of contemporary group of herd-visit, month and age of inspection, and lactation stage. The log-likelihood ratio test was used to determine if the genetic correlation between the measures of $M$. bovis infection and performance traits differed from zero.

Validation of Genetic Correlation Coefficients. Genetic correlations between $M$. bovis infection trait measures and performance traits were also estimated using threshold-linear sire models (which assumed an underlying distribution for the susceptibility to the $M$. bovis infection trait measures), with a logit link function, to determine if they were similar to those estimated using a linear-linear sire model. The $t$-test was used to test the difference among the correlation coefficients estimated between the $M$. bovis infection trait measures and performance traits from the linearlinear and threshold-linear models. Spearman rank correlations between breeding values for the same trait in the linear-linear and threshold-linear sire models were estimated to assess the ability of the linear-linear sire model to rank EBV compared with that of thresholdlinear sire model. The significance of the correlation coefficients was tested using the Fisher's Z-transform test, which compared the obtained coefficients to unity. Second, performance trait datasets were divided into 2 random subsets to determine the precision of the genetic correlation coefficients between the $M$. bovis infection trait measures and performance traits estimated from the linear-linear sire model compared with that of the threshold-linear sire model. The splitting technique used in this study involved a random partitioning of the complete dataset into 2 equally sized subsets by contemporary group. The $t$-test was used to test the difference between the correlation coefficients estimated from both validation sub-datasets. The precision (as measured by the mean squared error; Gates et al., 1999) of correlation coefficients estimated on the visible and underlying liability scale was compared.

Selection Index Methodology. Correlations among the $M$. bovis infection trait measures and performance traits were combined into genetic and phenotypic correlation matrices. An iterative bending procedure (Jorjani et al., 2003) was used to make the resulting matrices positive definite. When the degree of bend exceeded 1 standard error unit, bivariate analysis was rerun, and the likelihood ratio test between nested models was used to determine whether the bent correlation coefficient between the traits differed significantly from the original estimates. The breeding goal was to increase genetic resistance against confirmed $M$. bovis infection for a variety of scenarios, in which the relative importance of resistance and production traits was varied. Predicted correlated responses to selection in confirmed $M$. bovis infection were calculated in all cases. The response to selection for increased resistance to confirmed $M$. bovis infection was examined under 3 alternative indexes: (1) a performance index, herein referred to as Perform, which included traits that were genetically correlated with the $M$. bovis infection traits measures; (2) a PerformcMbo index, where confirmed M. bovis infection was included in the selection index, and (3) a PerformMboPPD responsiveness index, where $M$. bovis-PPD responsiveness was included as an indicator trait for confirmed $M$. bovis infection in the selection index. Economic values (approximately $€ 0.09 / \mathrm{kg}$ of milk yield, €0.96/kg of fat yield, €5.36/ kg of protein yield, approximately €55.48 per unit increase in SCS, approximately €10.87/d increase in CIN, and €10.51 per percent increase in survival per lactation) from Berry et al. (2009a) were used to estimate the relative emphasis of the performance traits in the Perform index. The economic weight on the performance traits in the Perform index was divided evenly across first to third parity. The proportional emphasis on traits within the Perform subindex was held constant while testing relative emphases ranging from 0 to $52 \%$ on confirmed $M$. bovis infection within the breeding goal.

\section{RESULTS}

The proportion of positive animals was 5.7 and $12.4 \%$ in the confirmed $M$. bovis infection and $M$. bovis-PPD responsiveness datasets, respectively. The heritability (SE of estimate in parentheses) from the linear sire model was $0.02(0.01)$ for confirmed $M$. bovis infection and 0.06 (0.02) for M. bovis-PPD responsiveness. Summary statistics and heritability estimates for all perfor- 
Table 1. Raw phenotypic means ( $\mu$; SD in parentheses) of first- to third-parity milk, fat, and protein production; SCC; calving interval (CIN); first-parity BCS; percentage survival from first to second, second to third, and third to fourth parity; and heritability estimates (SE in parentheses) from the linear sire model for all performance traits

\begin{tabular}{|c|c|c|c|c|c|c|}
\hline Trait & \multicolumn{2}{|c|}{ Parity 1} & \multicolumn{2}{|c|}{ Parity 2} & \multicolumn{2}{|c|}{ Parity 3} \\
\hline Milk, kg & $6,085(1,324)$ & $0.29(0.023)$ & $6,907(1,451)$ & $0.31(0.03)$ & $7,239(1,477)$ & $0.28(0.03)$ \\
\hline Protein, kg & $203(42)$ & $0.27(0.02)$ & $235(46)$ & $0.29(0.02)$ & $246(47)$ & $0.25(0.03)$ \\
\hline $\mathrm{SCC}$, cells $/ \mu \mathrm{L}$ & $128(209)$ & $0.11(0.01)$ & $144(231)$ & $0.13(0.02)$ & $175(265)$ & $0.10(0.02)$ \\
\hline CIN, d & $404(84)$ & $0.03(0.01)$ & $398(75)$ & $0.03(0.01)$ & $393(71)$ & $0.03(0.01)$ \\
\hline
\end{tabular}

mance traits investigated in this study are presented in Table 1.

\section{Genetic Parameters}

Genetic correlations between the $M$. bovis infection trait measures and performance traits estimated using linear-linear sire models are summarized in Table 2. In general, a positive genetic association existed among the $M$. bovis infection trait measures and the milk production traits, suggesting that increased production is associated with increased susceptibility to $M$. bovis infection. Nonetheless, only second-parity fat production was significantly genetically correlated with $M$. bovis-PPD responsiveness. The $M$. bovis infection trait measures were negatively genetically associated with SCS and CIN or genetic correlations were close to 0 , suggesting that reduced SCS and CIN are associated with increased susceptibility to M. bovis infection. However, only first-parity SCS was significantly genetically correlated with $M$. bovis-PPD responsiveness. Negative genetic associations were found in the main between the $M$. bovis infection trait measures and survival, suggesting that increased survival is associated with increased resistance to $M$. bovis infection. However, only the genetic correlation between $M$. bovis-PPD responsiveness and survival from third to fourth parity differed significantly from zero. A positive genetic association existed between the $M$. bovis infection trait measures and first-parity BCS, suggesting increased BCS is associated with increased susceptibility to $M$. bovis infection. However, only the genetic correlation between $M$. bovis-PPD responsiveness and BCS was significantly different from zero.

Genetic correlations estimated using the linear-linear sire model between $M$. bovis-PPD responsiveness and the performance traits that were significantly different from 0 were similar to, and equally or more precise than, those estimated using the threshold-linear sire model (Table 3). Sire ranking of the linear-linear model was similar to that of the threshold-linear sire model. Spearman's rank correlations among sire estimated EBV for all traits estimated from the linear-linear and threshold-linear sire models ranged from 0.85 to $0.98(P<0.05 ;$ significance of difference from 1$)$. The phenotypic correlations among the $M$. bovis infection trait measures and the performance traits tended to be weaker than their corresponding genetic correlations, although the signs of the correlations were generally the same.

Table 2. Genetic correlations (SE in parentheses) between confirmed Mycobacterium bovis infection and the performance traits first- to thirdparity 305-d milk, fat, and protein production; percentage fat and protein; SCS; calving interval (CIN); survival from first to second, second to third, and third to fourth parity; and first-parity BCS estimated using the sire linear-linear models ${ }^{1}$

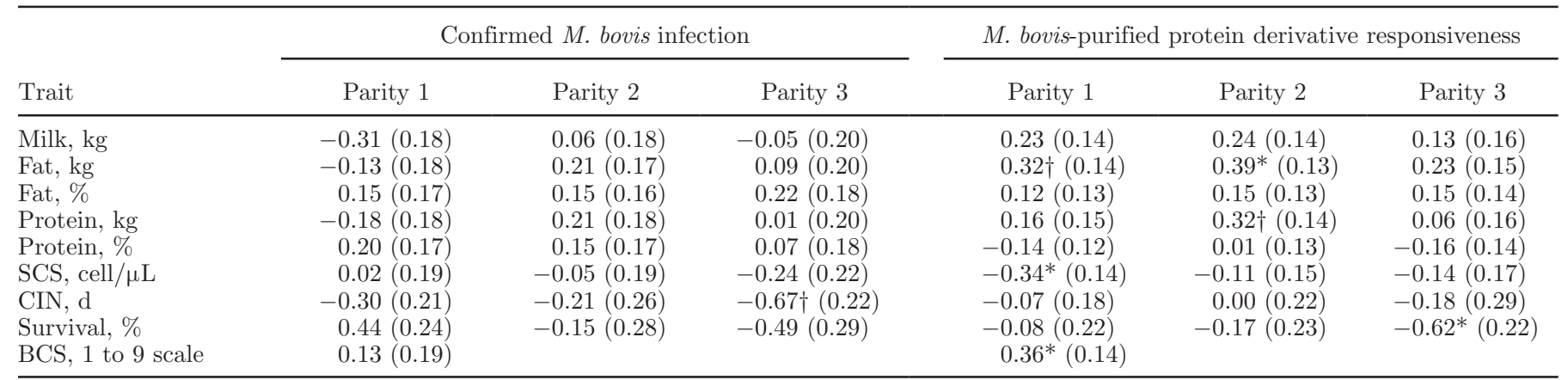

${ }^{1}$ Significance of difference from zero: $\dagger P<0.10 ;{ }^{*} P<0.05$. 
Table 3. Genetic correlations (SE in parentheses) between Mycobacterium bovis-purified protein derivative (PPD) responsiveness and the performance traits second-parity 305-d fat production, first-parity SCS, survival from third to fourth parity, and first-parity BCS from the sire linear-linear and threshold-linear models (LLM and TLM, respectively) from the original (SE significance if inter-model comparison) and split-validation datasets (SE significance if intra-model comparison) 1 and 2 (Val. 1 and Val. 2, respectively), and mean square error (MSE) of the LLM and TLM ${ }^{1}$

\begin{tabular}{|c|c|c|c|c|}
\hline \multirow[b]{2}{*}{ Item } & \multicolumn{4}{|c|}{ M. bovis-PPD responsiveness } \\
\hline & Original & Val. 1 & Val. 2 & MSE \\
\hline \multicolumn{5}{|l|}{ Fat } \\
\hline LLM & $0.39(0.13)$ & $0.20 \ddagger(0.14)$ & $0.37 \ddagger(0.12)$ & 0.023 \\
\hline TLM & $0.37 \ddagger(0.13)$ & $0.12 \ddagger(0.13)$ & $0.42 \ddagger(0.12)$ & 0.021 \\
\hline \multicolumn{5}{|l|}{ SCS } \\
\hline LLM & $-0.34(0.14)$ & $-0.14 \ddagger(0.15)$ & $-0.23 \ddagger(0.14)$ & 0.049 \\
\hline TLM & $-0.29 \ddagger(0.14)$ & $-0.09 \ddagger(0.14)$ & $-0.18 \ddagger(0.14)$ & 0.049 \\
\hline \multicolumn{5}{|c|}{ Survival } \\
\hline LLM & $-0.62(0.22)$ & $-0.81 \ddagger(0.11)$ & $-0.38 \ddagger(0.15)$ & 0.001 \\
\hline TLM & $-0.67 \ddagger(0.21)$ & $-0.86 \ddagger(0.19)$ & $-0.35 \ddagger(0.16)$ & 0.008 \\
\hline \multicolumn{5}{|l|}{ BCS } \\
\hline LLM & $0.36(0.14)$ & $0.24 \ddagger(0.14)$ & $0.26 \ddagger(0.14)$ & 0.025 \\
\hline TLM & $0.35 \ddagger(0.13)$ & $0.21 \ddagger(0.13)$ & $0.26 \ddagger(0.14)$ & 0.026 \\
\hline
\end{tabular}

${ }^{1}$ Significance was based on the $t$-test, which tested whether genetic correlations estimated from the LLM and TLM differed significantly from one another, and whether genetic correlations estimated from the validation datasets differed significantly from the original estimate; $\ddagger P>0.05$.

The correlation matrix derived following the weighted bending procedure is presented in Table 4. Following the bending process, out of 132 phenotypic and genetic correlations, 70 correlations changed by less than 0.05 standard error units, and 112 correlations changed by less than 0.50 standard error units from their original estimate. One correlation changed more than 1 standard error unit from its original estimate. Nevertheless, the bent correlation coefficient was not significantly different from the original estimate $(P>0.05)$.

\section{Predicted Response to Selection Indexes}

The following traits were added as goal and index traits in all 3 indices: first- to third-parity fat yield and SCS, and survival from first to second, second to third, and third to fourth parity. Body condition score was also added as an index trait in the 3 indices. The absence of emphasis on confirmed $M$. bovis infection within the Perform index resulted in a genetic response per generation of increased susceptibility to confirmed

Table 4. Genetic (below diagonal) and phenotypic (above diagonal) correlation matrix of Mycobacterium bovis infection trait measures: confirmed M. bovis infection (cMbo) and M. bovis-purified protein derivative responsiveness (PPD), and genetically correlated performance traits $(P<0.05)$ : first- to third-parity 305-d fat production and SCS, survival from first to second, second to third and third to fourth parity, and first-parity BCS from the linear-linear analysis following the weighted bending procedure (heritabilities on diagonal)

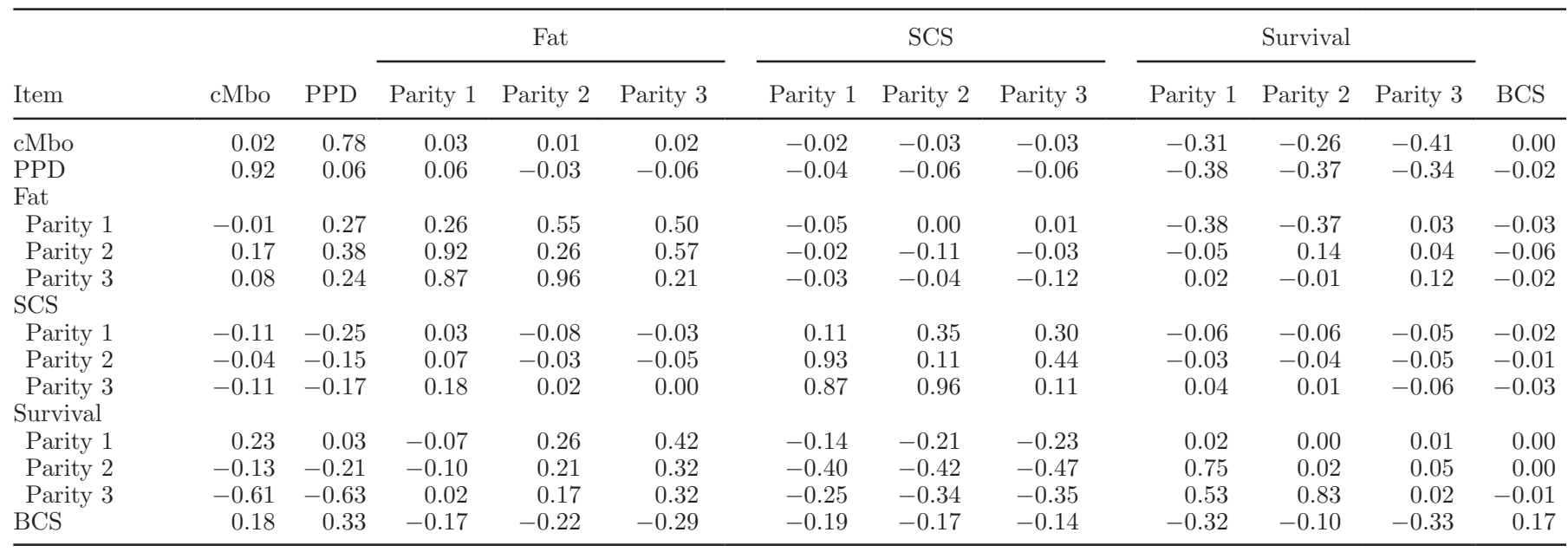




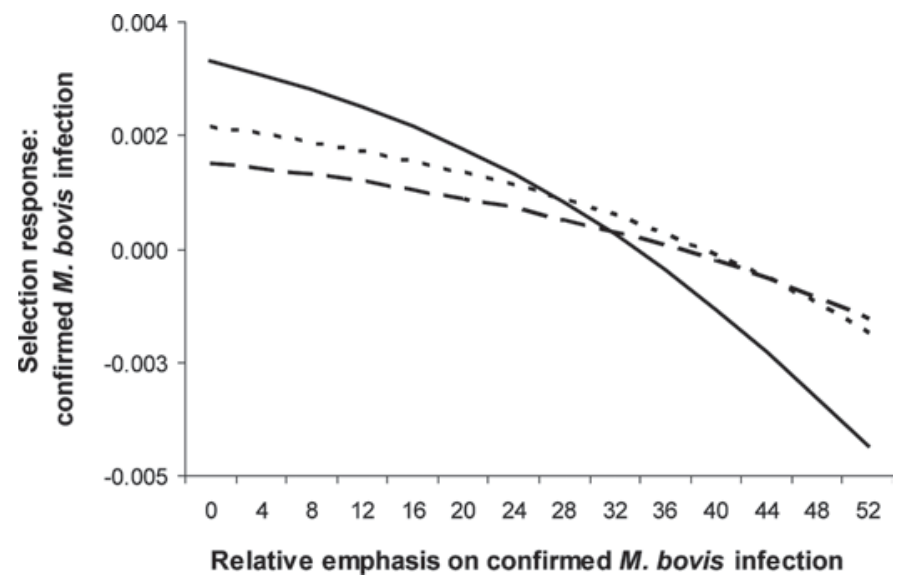

Figure 1. The response to selection for susceptibility to confirmed Mycobacterium bovis infection using the Perform index (dashed lines), which included the index traits second-parity fat yield, third-parity SCS, survival from third to fourth parity, and first-parity BCS; the PerformcMbo index (dotted line), where confirmed $M$. bovis infection was included in the selection index; and the PerformMboPPD responsiveness index (solid line), where M. bovis-purified protein derivative responsiveness was included as an indicator trait for confirmed $M$. bovis infection in the selection index, with varying relative emphasis on confirmed $M$. bovis infection within the breeding goal.

$M$. bovis infection by $0.1 \%$ per standardized selection differential (Figure 1). A relative emphasis on confirmed $M$. bovis infection within the breeding goal of $32 \%$ in the Perform and PerformMboPDD indices and $36 \%$ in the PerformcMbo index was required to halt the antagonistic effect of selecting on improved performance on genetic response in susceptibility to confirmed $M$. bovis infection. The Perform and PerformcMbo indices at a relative emphasis of $40 \%$, and PerformMboPDD

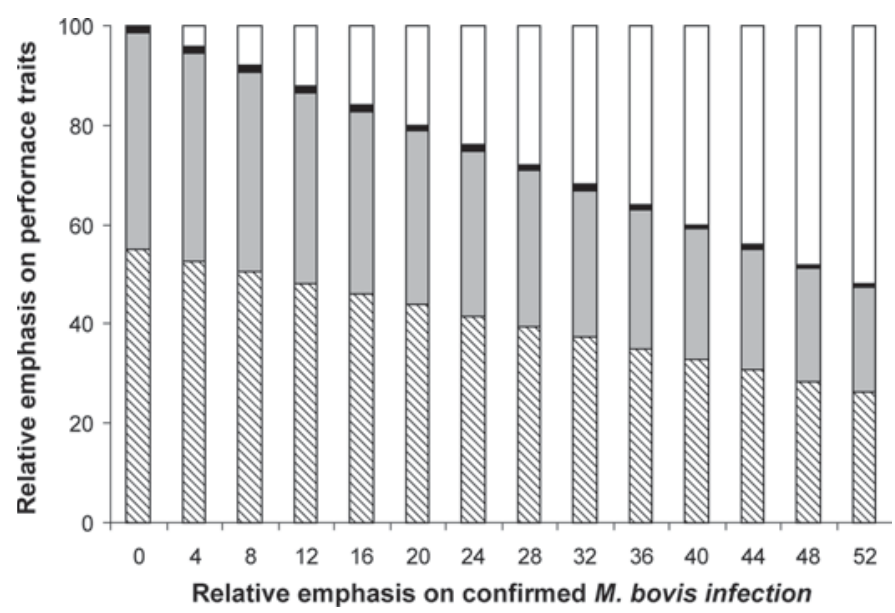

Figure 2. The varying relative emphases applied to confirmed Mycobacterium bovis infection (white), survival (black), SCS (gray), and fat production (diagonal lines) within the breeding goal with varying relative emphasis on confirmed $M$. bovis infection within the breeding goal. index at a relative emphasis of $36 \%$ on confirmed $M$. bovis infection within the breeding goal, resulted in a $0.1 \%$ reduction per generation in susceptibility to confirmed $M$. bovis infection per standardized selection differential. However, as the relative emphasis on confirmed $M$. bovis infection was increased, there was an equalized reduction of the relative emphasis placed on performance traits within the breeding goal (Figure 2 ). The Perform and PerformcMbo indices at a relative emphasis of $40 \%$, and the PerformMboPDD index at a relative emphasis of $36 \%$ on confirmed $M$. bovis infection within the breeding goal, resulted in a decrease in the rate of economic gain of $€ 0.23, € 0.19$, and $€ 0.25$ per cow per standardized selection differential in the Perform, PerformcMbo, and PerformMboPDD indices, respectively.

\section{DISCUSSION}

The presence of ample genetic variation reported previously for confirmed $M$. bovis infection (Bermingham et al., 2009) indicates that genetic selection for increased resistance to $M$. bovis infection is possible. However, nothing is known about the genetic correlations between susceptibility to $M$. bovis infection and economically important traits in lactating dairy cows. The objective of this study was to estimate the genetic associations between measures of $M$. bovis infection and economically important traits and to determine the effect of selection for increased performance on genetic response to resistance to $M$. bovis infection. The heritability estimates for susceptibility to confirmed $M$. bovis infection and $M$. bovis-PPD responsiveness were similar to those reported by Bermingham et al. (2009), although the present study contained 46 and $23 \%$ more records for confirmed $M$. bovis infection and M. bovisPPD responsiveness, respectively. Estimates of heritability for all performance traits in this study are within the range of estimates from the literature (Ingvartsen et al., 2003; Tsuruta et al., 2005).

The positive genetic correlation between $M$. bovisPPD responsiveness and second-parity fat yield is the same as that reported between clinical mastitis and milk yield (Ingvartsen et al., 2003; Carlén et al., 2004; Negussie et al., 2008). Berry et al. (2009b) reported to the contrary, a negative genetic correlation between the AMIR to $M$. avium sub. paratuberculosis and secondand third-parity fat yield. High AMIR in M. bovis-infected cattle is associated with anergy of the CMIR and disseminated disease (Welsh et al., 2005). The AMIR in Berry et al. (2009b) may therefore have provided an indirect measure of the efficacy of the CMIR against M. avium ssp. paratuberculosis infection. Susceptibility to confirmed $M$. bovis infection, which is a measure of 
both the efficacy of the innate immune response and CMIR against $M$. bovis infection, was not genetically correlated with production in this study. It is possible that antagonistic associations between production and the innate immune response and CMIR may have counteracted each other, resulting in a genetic correlation between susceptibility to confirmed $M$. bovis infection and production near zero. The negative genetic correlation between $M$. bovis-PPD responsiveness and firstparity SCS is in agreement with a recent phenotypic study, which demonstrated $M$. bovis-PPD responders that survived to the fifth parity had lower SCC than did nonresponders (Boland et al., 2010). On the other hand, estimates of genetic correlations among SCS, mastitis, and production from the literature are positive (Carlén et al., 2004; Negussie et al., 2008). The favorable association between SCS and M. bovis-PPD responsiveness in this study may therefore support antagonistic genetic effects linked to phases of immune response producing genetic associations between susceptibility to confirmed $M$. bovis infection and production traits close to zero. A strong negative genetic association was estimated between survival from third to fourth parity and susceptibility to $M$. bovis-PPD responsiveness; this indicated that cows with high genetic merit for survival are genetically less disposed to becoming a standard reactor following exposure to $M$. bovis. This is in agreement with Heringstad et al. (2003), who reported a positive genetic correlation between risk of culling and both clinical mastitis and SCC.

A modest positive genetic correlation also existed between $M$. bovis-PPD responsiveness and BCS in this study. This is in line with Lassen et al. (2003), who reported an unfavorable genetic association between health and BCS. However, the same authors reported a negative genetic correlation between BCS and mastitis (Lassen et al., 2003). Genetic correlations reported between BCS and disease are inconsistent (Lassen et al., 2003; Dechow et al., 2004), which may be indicative of an intermediate optimum for BCS and cow health, and hence a nonlinear relationship between BCS and susceptibility to $M$. bovis-PPD responsiveness.

The $M$. bovis infection trait measures were not genetically correlated with milk yield. A phenotypic study on Irish data has however recently shown that $M$. bovisPPD responders produced significantly less milk yield than nonreactors in all lactations (Boland et al., 2010). However, genetic and phenotypic correlations with opposing signs have been reported, where positive genetic and negative phenotypic associations were estimated between milk yield and SCC (Haile-Mariam et al., 2001; Koivula et al., 2005). A recent United Kingdombased study has nevertheless documented a favorable genetic correlation between resistance to TB and milk yield (Brotherstone et al., 2010). The different results from the United Kingdom and Ireland may have been a result of differences in genetic background of respective cow populations, or previous selection or TB eradication practices that may have unconsciously favored particular clinical or nonclinical phenotypes (and hence genotypes). Furthermore, differential diagnostic accuracy of SICTT in the United Kingdom and Ireland may provide an alternative explanation for the differences between the 2 studies. The SICTT testing intervals in the United Kingdom are dependent on the prevalence of bovine TB (Collins, 2006), hypothetically resulting in more variable diagnostic accuracy compared with the mandatory annual intervals Ireland (More and Good, 2006). The use of data from more regularly tested contiguous herd breakdowns from UK SICTT data may reduce the supposed differential in diagnostic accuracy between the UK and Irish data. The inconsistencies between the UK and Irish studies need to be resolved before any definitive conclusions can be drawn for the dairy industry. Future work that replicates the methodologies used in this study in the United Kingdom and on independent Irish data are needed to corroborate our findings.

\section{Methodological Issues}

All major effects associated with risk of failing the SICTT (More and Good, 2006) and animal performance (Berry et al., 2009a,b) were accounted for in the data editing or adjusted for in the statistical models. A substantial attempt was also made to maximize the opportunity of exposure to $M$. bovis between and within herds to facilitate the partitioning of genetic from environmental variance (Bermingham et al., 2009; Brotherstone et al., 2010). Furthermore, a high diagnostic threshold to detect standard $M$. bovis reactors was set, and inconclusive reactors were removed from the analysis of $M$. bovis-PPD responsiveness to reduce the likelihood of false positives or negatives.

Several studies have considered the binary nature of functional traits and have demonstrated that thresholdlinear models perform better than linear-linear models (Van Vleck and Gregory, 1992; Varona et al., 1999; Gates et al., 1999). In this study, the genetic correlations between the measures of $M$. bovis infection and the performance traits from the linear-linear sire model was similar to, and equally or more precise than, those from the threshold-linear sire model. Two earlier studies (Heringstad et al., 2005; Negussie et al., 2008) have also reported similar genetic correlations from linear-linear and threshold-linear models. The sire rankings from the linear-linear and threshold-linear sire model were similar. This may have resulted from the medium- to 
large-sized paternal half-sib groups used in this study; it has been reported that as the number of progeny per sire increases, differences between the models decrease (Heringstad et al., 2003, 2005; Negussie et al., 2008). The amount of available information per sire in national genetic evaluations is generally large; computationally less intensive linear-linear models may therefore be preferentially implemented for the estimation of (co) variance components for susceptibility to the $M$. bovis infection trait measures.

\section{Implications for Genetic Improvement}

Selection for increased survival may indirectly reduce susceptibility to $M$. bovis infection, whereas selection for reduced SCS and increased fat production and BCS may increase susceptibility to $M$. bovis infection within the national Holstein-Friesian dairy herd. The greater amount of genetic variation in $M$. bovis-PPD responsiveness allowed for larger contribution within the selection index, and hence indirect contribution of confirmed $M$. bovis infection within the breeding goal, facilitating the augmented response to selection observed in the PerformMboPDD index. A $0.1 \%$ reduction in susceptibility to confirmed $M$. bovis infection at an emphasis of $36 \%$ within the breeding goal may seem a slow rate of change, but a $0.1 \%$ reduction in susceptibility to confirmed $M$. bovis infection at a $M$. bovis-PPD reactor incidence of $0.33 \%$ (Good et al., 2007 ) and cost per reactor of $€ 1,018$ (Sheppard and Turner, 2005) would provide an economic gain of $€ 210$ in a 100-cow herd. The high level of emphasis required to reduce susceptibility to confirmed $M$. bovis infection will, however, deflect the emphasis from economically important traits. The results of this study indicate that a breeding goal with moderate emphasis on confirmed $M$. bovis infection, to reduce or halt the antagonistic impact of genetic selection for increased genetic merit for economically important traits, may therefore be more applicable in Irish dairy cattle breeding programs. This national breeding goal could be coupled with tailored herd-specific breeding strategies, with an emphasis on confirmed $M$. bovis infection weighted by prevailing incidence of TB relative to individual herd production goals. Nevertheless, before any definitive conclusions can be drawn, a full economic analysis of the cost of bovine TB is required to determine the optimal emphasis on confirmed $M$. bovis infection in selection indices relative to other traits and to quantify economic benefits from consideration of confirmed $M$. bovis infection in breeding programs.

\section{CONCLUSIONS}

The results of this study based on large datasets and alternative statistical approaches suggest that selection for increased survival may indirectly reduce susceptibility to $M$. bovis infection, whereas selection for reduced SCS and increased fat production and BCS may increase susceptibility to $M$. bovis infection. Current selection strategies are therefore likely to lead to a marginal increase in susceptibility to $M$. bovis infection within the national Holstein-Friesian dairy herd. Indirect selection on the indicator trait measure, $M$. bovis-PPD responsiveness, within the selection index produced a greater response to selection than did direct selection on confirmed $M$. bovis infection. Nonetheless, substantial emphasis on confirmed $M$. bovis infection within the breeding goal at the expense of performance was required to increase resistance against confirmed $M$. bovis infection. A national breeding goal to halt the negative effects of resistance to confirmed $M$. bovis infection associated with selection for increased genetic merit for economically important traits, coupled with strategic herd-specific breeding goals to increase resistance to confirmed $M$. bovis infection, may therefore be more applicable.

\section{ACKNOWLEDGMENTS}

Financial support from the Department of Agriculture, Fisheries and Forestry Eradication of Animal Disease Board (Dublin, Ireland) is gratefully acknowledged.

\section{REFERENCES}

Akbas, Y., S. Brotherstone, and W. G. Hill. 1993. Animal model estimation of non-additive genetic parameters in dairy cattle, and their effect on heritability estimation and breeding value prediction. J. Anim. Breed. Genet. 110:105-113.

Bermingham, M. L., S. J. More, M. Good, A. R. Cromie, I. M. Higgins, S. Brotherstone, and D. P. Berry. 2009. Genetics of tuberculosis in Irish Holstein-Friesian dairy herds. J. Dairy Sci. 92:3447-3456.

Berry, D., L. Shalloo, A. Cromie, V. Olori, R. Veerkampc, P. Dillon, P. Am, R. Evans, F. Kearney, and B. Wickham. 2009a. The economic breeding index: A generation on. Technical report to the Irish Cattle Breeding Federation. http://www.icbf.com/publications/files/ economic_breeding_index.pdf. Accessed Mar. 2, 2007.

Berry, D. P., M. Good, P. Mullowney, A. R. Cromie, and S. J. More. 2009b. Genetic variation in serological response to Mycobacterium avium subspecies paratuberculosis and its association with performance in Irish Holstein-Friesian dairy cows. Livest. Sci. 131:102107.

Boland, F., G. E. Kelly, M. Good, and S. J. More. 2010. Bovine tuberculosis and milk production in infected dairy herds in Ireland. Prev. Vet. Med. 93:153-161.

Brotherstone, S., I. M. S. White, M. Coffey, S. H. Downs, A. P. Mitchell, R. S. Clifton-Hadley, S. J. More, M. Good, and J. A. Wool- 
liams. 2010. Evidence of genetic resistance of cattle to infection with Mycobacterium bovis. J. Dairy Sci. 93:1234-1242.

Carlén, E., E. Strandberg, and A. Roth. 2004. Genetic parameters for clinical mastitis, somatic cell score and production in the first three lactations of Swedish Holstein cows. J. Dairy Sci. 87:3062-3070.

Collins, J. D. 2006. Tuberculosis in cattle: Strategic planning for the future. Vet. Microbiol. 112:369-381.

Corner, L., L. Melville, K. McCubbin, K. J. Small, B. S. McCormick, P. R. Wood, and J. S. Rothel. 1990. Efficiency of inspection procedures for detection of tuberculosis lesions in cattle. Aust. Vet. J. $67: 389-392$.

Crump, R. E., N. R. Wray, R. Thompson, and G. Simm. 1997. Assigning pedigree beef performance records to contemporary groups taking account of within-herd calving patterns. Anim. Sci. 65:193-198.

Dechow, C. D., G. W. Rogers, U. Sander-Nielsen, L. Klei, T. J. Lawlor, J. S. Clay, A. E. Freeman, G. Abdel-Azim, A. Kuck, and S. Schnell. 2004. Correlations among body condition scores from various sources, dairy form, and cow health from the United States and Denmark. J. Dairy Sci. 87:3526-3533.

Gates, P., K. Johansson, and B. Danell. 1999. "Quasi-REML" correlation estimates between production and health traits in presence of selection and confounding: A simulation study. J. Anim. Sci. $77: 558-568$.

Gilmour, A. R., B. R. Cullis, S. J. Welham, and R. Tompson. 2009. ASREML reference manual. New South Wales Agriculture, Orange Agricultural Institute, Orange, Australia.

Good, M., I. Higgins, and P. Maher. 2007. The tuberculin test-A safe means to test a cattle population for bovine tuberculosis. Ir. Vet. J. 60:680-684.

Haile-Mariam, M., P. J. Bowman, and M. E. Goddard. 2001. Genetic and environmental correlations between test-day somatic cell count and milk yield traits. Livest. Prod. Sci. 73:1-13.

Heringstad, B., Y. M. Chang, D. Gianola, and G. Klemetsdal. 2005. Genetic association between susceptibility to clinical mastitis and protein yield in Norwegian dairy cattle. J. Dairy Sci. 88:15091514.

Heringstad, B., R. Rekaya, D. Gianola, G. Klemetsdal, and K. A. Weigel. 2003. Bivariate analysis of liability to clinical mastitis and to culling in first lactation cows. J. Dairy Sci. 86:653-660.

Ingvartsen, K. L., R. J. Dewhurst, and N. C. Friggens. 2003. On the relationship between lactational performance and health: Is it yield or metabolic imbalance that cause production diseases in dairy cattle? A position paper. Livest. Prod. Sci. 83:277-308.

Jorjani, H., L. Klei, and U. Emanuelson. 2003. A simple method for weighted bending of genetic (co)variance matrices. J. Dairy Sci. 86:677-679.

Koivula, M., E. A. Mantyssaari, E. Negussie, and T. Serenius. 2005. Genetic and phenotypic relationships among milk yield and somatic cell count before and after clinical mastitis. J. Dairy Sci. 88:827-833.

Lassen, J., M. Hansen, M. K. Sørensen, G. P. Aamand, L. G. Christensen, and P. Madsen. 2003. Genetic relationship between body condition score, dairy character, mastitis, and diseases other than mastitis in first-parity Danish Holstein cows. J. Dairy Sci. 86:3730-3735.

More, S. J., and M. Good. 2006. The tuberculosis eradication programme in Ireland: A review of scientific and policy advances since 1988. Vet. Microbiol. 112:239-251.

Negussie, E., I. Strandén, and E. A. Mäntysaari. 2008. Genetic analysis of liability to clinical mastitis, with somatic cell score and production traits using bivariate threshold-linear and linear-linear models. Livest. Sci. 117:52-59.

Rupp, R., A. Hernandez, and B. A. Mallard. 2007. Association of bovine leukocyte antigen (BoLA) DRB3.2 with immune response, mastitis, and production and type traits in Canadian Holsteins. J. Dairy Sci. 90:1029-1038.

Sheppard, A., and M. Turner. 2005. An economic impact assessment of bovine tuberculosis in southwest England. Research Report for the South West of England Regional Development Agency by the Uni- versity of Exeter Centre for Rural Research, Devon, UK. http:// purl.umn.edu/31748 Accessed Jun. 6, 2009.

Thoen, C. O., and B. R. Bloom. 1995. Pathogenesis of Mycobacterium bovis. Pages 3-14 in Mycobacterium bovis Infection in Animals and Humans. C. O. Thoen and J. H. Steele, ed. Iowa State University Press, Ames.

Tsuruta, S., I. Misztal, and T. J. Lawlor. 2005. Changing definition of productive life in US Holsteins: Effect on genetic correlations. J. Dairy Sci. 88:1156-1165.

Van Vleck, L. D., and K. E. Gregory. 1992. Multiple-trait restricted maximum likelihood for simulated measures of ovulation rate with underlying multivariate normal distributions. J. Anim. Sci. 70:57-61.

Varona, L., I. Misztal, and J. K. Bertrand. 1999. Threshold-linear versus linear-linear analysis of birth weight and calving ease using an animal model: II. Comparison of models. J. Anim. Sci. 77:2003-2007.

Welsh, M. D., R. T. Cunningham, D. M. Corbett, R. M. Girvin, J. McNair, R. A. Skuce, D. G. Bryson, and J. M. Pollock. 2005. Influence of pathological progression on the balance between cellular and humoral immune responses in bovine tuberculosis. Immunology 114:101-111.

\section{APPENDIX}

\section{Mycobacterium bovis Infection Trait Measures}

A total of 2,094,750 animal SICTT results from 17,213 positive herd-tests (i.e., herd-tests with at least $1 \mathrm{M}$. bovis standard reactor) in 5,905 herds and 21,889 positive abattoir animal lesion records from November 2000 to December 2007 were obtained from the Department of Agriculture, Fisheries and Forestry's Animal Health Computer System and the Factory Lesion Database, respectively. Movements $(\mathrm{n}=720,960)$ of all animals in and out of herds are recorded in Ireland and dates of movements as well as herd of origin and destination were obtained on all animal movements from January 2000 to December 2007 from the Department of Agriculture, Fisheries and Forestry's Cattle Movement and Monitoring System. Calving records and pedigree information were obtained from the Irish Cattle Breeding Federation database.

Atypical herd-tests (i.e., herd-tests with more than $5 \mathrm{M}$. bovis standard reactors but no observed factory lesion disclosed) were deleted as described by Bermingham et al. (2009). The SICTT records on 1,205,318 animals (33,633 M. bovis standard reactors) across 9,395 herd-tests from 4,238 herds remained ( $62 \%$ of herds had $1,19 \%$ had $2,7 \%$ had $3,3 \%$ had 4 , and $9 \%$ had 5 or more positive herd-tests).

Mycobacterium bovis-PPD Responsiveness Dataset. A total of 246,868 animals with calving records from 2,461 herds (5,563 positive herd-tests) within 3,276 episodes (i.e., herd restrictions initiated by 2 or more $M$. bovis standard reactors and terminated by 2 consecutive clear herd-tests; Bermingham et al., 2009) were retained. Parity number was recoded into 6 
classes: $1,2,3,4,5$, and $>5$. Cows of unknown parity and less than 15 mo old, and heifers calving within 6 wk after the herd-test were discarded $(\mathrm{n}=954)$. Cows with an age at calving greater than 22 mo from the parity median or that were less than $75 \%$ Holstein-Friesian were removed $(\mathrm{n}=24,594)$. Cows within episodes that lacked a home-bred $M$. bovis standard reactor, nonreactor cows with subsequent inconclusive SICTT or abattoir lesion records, cows that moved into the herd within 6 wk of the herd test, inconclusive $M$. bovis reactors, $M$. avium reactors, and cows without a known sire were discarded $(\mathrm{n}=183,285)$. The last record per cow within the dataset was retained. An iterative algorithm was invoked to remove cows from paternal half-sib groups with less than 4 progeny, episodes with no standard $M$. bovis reactor, and episodes with fewer than 10 cows $(\mathrm{n}=6,668)$.

Confirmed $M$. bovis Infection Dataset. A total of 157,340 animals with calving records from 1,657 herds (3,255 positive herd-tests) within 2,040 episodes were retained. The criterion of a home-bred TB case within each episode as described by Bermingham et al. (2009) was not implemented as this requirement resulted in excessive loss of TB case records when generating a dataset for the analysis of confirmed $M$. bovis infection. Nonlesioned animals with standard or inconclusive SICTT results $(\mathrm{n}=41,799)$ were deleted. Editing criteria were otherwise the same as those for the SICTT data. An iterative algorithm was invoked to remove cows from paternal groups with fewer than 4 half-sibs $(\mathrm{n}=2,631)$, episodes with no lesioned animals, and episodes with fewer than 10 cows $(\mathrm{n}=1,228$; 22,177 cow records).

\section{Performance Traits Data Edits}

First- to third-parity 305-d milk, fat, and protein yield; SCC ( $\mathrm{n}=3,642,006)$; calving date; CIN ( $\mathrm{n}=$ $6,495,139)$; and first-parity $\mathrm{BCS}(\mathrm{n}=86,814)$ records were extracted from the Irish Cattle Breeding Federation database for cows calving between January 1985 and December 2007. Survival from lactation 1 to 2, 2 to 3 , and 3 to 4 was obtained for each cow. If an animal had a second calving date recorded, then it was assumed to have survived to second lactation; a similar criterion was used to determine if the cow had survived to later parities. Cows with death or culling records in a particular lactation were assumed not to have survived to the next lactation. If the last recorded date of a cow was a noncalving date (e.g., milk test-day record), and that record was more than $140 \mathrm{~d}$ before the last known milk-recording date in the herd, then it was assumed to have been culled in that lactation. If the last recorded date was within $140 \mathrm{~d}$ of the last known herd milk-recording date, then it was coded as missing. If the last recorded date of a cow was its calving date, then it was assumed culled if this was more than $800 \mathrm{~d}$ from the last known herd date; otherwise it was coded as missing. This edit accounted for herds that did not record death or culling events or did not participate in milk recording.

Cows with CIN less than 300 or greater than 800 d, cows of unknown parity and less than 15 mo old, and cows with an age at calving greater than 22 mo from the parity median were removed. Animals less than $75 \%$ Holstein-Friesian or with no identified sire were discarded. Contemporary groups for herd-yearseason calving were formed using algorithms outlined by Crump et al. (1997). Initially, calving dates differing by $10 \mathrm{~d}$ were placed in separate contemporary groups. Subsequently, if the number of records within any contemporary group was less than 8 , the contemporary group was merged with a contemporary group adjacent in time if the start and end dates of the adjacent contemporary groups were less than 31 d. Subsequently, only contemporary groups with at least 5 animals were retained. A random selection of herd data was retained to maintain a dataset size below 100,000 records to facilitate computing. Herd-visit contemporary groups for BCS were derived by concatenating herd and date of visit. Finally, an iterative algorithm was used to remove any contemporary group with less than 5 records and paternal half sib group of fewer than 5 progeny records.

\section{Heterosis and Recombination Loss}

Heterosis and recombination loss were calculated for each animal as follows (Akbas et al., 1993):

$$
\begin{gathered}
\text { Heterosis }=\mathrm{P}_{\mathrm{S}}\left(1-\mathrm{P}_{\mathrm{D}}\right)+\mathrm{P}_{\mathrm{D}}\left(1-\mathrm{P}_{\mathrm{S}}\right), \\
\text { Recombination loss }=\mathrm{P}_{\mathrm{D}}\left(1-\mathrm{P}_{\mathrm{D}}\right)+\mathrm{P}_{\mathrm{S}}\left(1-\mathrm{P}_{\mathrm{S}}\right),
\end{gathered}
$$

where $\mathrm{P}_{\mathrm{S}}$ and $\mathrm{P}_{\mathrm{D}}$ are the proportion of Holstein for the sire and dam, respectively. 